\title{
Electropolymerization and Multifunctional Properties of Novel Polypyrrole Films Embedded with Co Nanoparticles
}

\author{
Min Gao ${ }^{\mathrm{b}}$, Liying $\mathrm{Lu}^{\mathrm{a}, *}$, Xiaoguang $\mathrm{Xu}^{\mathrm{b}}$, Zhe Yao ${ }^{\mathrm{a}}$, Yong Jiang ${ }^{\mathrm{b}}{ }^{\mathrm{w}}$
}

\begin{abstract}
A novel electropolymerization process has been presented to prepare compact polypyrrole (PPy) polymer films embedded with different cobalt nanoparticle (Co NP) loadings by using cyclic voltammetry. An electropolymerization mechanism is suggested and further confirmed by Fourier transform infrared (FT-IR) spectroscopy and Raman spectroscopy of pure PPy and PPy polymer nanocomposites (PNCs). The PPy/Co PNCs exhibit unique multifunctional properties. Firstly, they are superparamagnets at room temperature, whose saturation magnetization can be controlled by modifying the concentration of Co NPs in the electrolyte solution. Secondly, they show not only semiconductor transportation behavior but also a negative magnetoresistance under external magnetic field. The permittivity of the PPy/Co PNCs switches from negative to positive with the increasing frequency. Both the outstanding magnetic and electrical properties make the PPy PNCs promising as one kind of microwave absorbing materials.
\end{abstract}

Key words: polypyrrole films, electropolymerization, magnetic property, dielectricity 


\section{Introduction}

Nowadays polymer nanocomposites (PNCs) prepared by dispersing magnetic nanoparticles (NPs) into a conducting polymer are proposed to have wide applications because of their many advantages, such as low cost, environmental stability, light weight, and unique electromagnetic properties ${ }^{[1-4]}$. Among diverse conducting polymers, polypyrrole (PPy) has attracted great attention due to its tunable conductivity by various ways of doping, easy preparation under different conditions, and outstanding pseudocapacitance as electrode materials ${ }^{[5-8]}$. In the past decades, PPy kept on the hottest research topics list and has already been used as microwave absorbing materials, adsorption materials, electrocatalytic reagents, magnetic-controlled switches, biomedical materials, biosensors, and corrosion inhibitors ${ }^{[9-15]}$.

PPy PNCs could get intrinsic magnetic properties by controlling their molecular structure by directly adding dopants consisting of iron species during polymerization ${ }^{[6,7]}$. However, this kind of intrinsic magnetism is very weak and hardly meets practical applications. PPy PNCs can also obtain external magnetic properties through compositing with magnetic metal or alloy crystal additives. For this kind of PPy PNCs, the magnetic properties become reasonably better, and thus has been extensively studied ${ }^{[9,16-19]}$. The magnetic additives are normally focused on $\mathrm{Ni}, \mathrm{Fe}$, $\mathrm{Fe}_{3} \mathrm{O}_{4}, \gamma-\mathrm{Fe}_{2} \mathrm{O}_{3}, \mathrm{BaFe}_{12} \mathrm{O}_{19}{ }^{[9,16-19]}$, etc. However, there are only a very few reports about PPy PNCs doped with cobalt (Co), even though Co is a very classical magnetic metal.

Face-centered-cubic (fcc) Co has a very high saturation (166 emu/g) being much larger than $\mathrm{Fe}_{3} \mathrm{O}_{4}(97 \mathrm{emu} / \mathrm{g})$ which is one of the most common magnetic materials in practice ${ }^{[20]}$. While reducing their size, the saturation magnetization of Co NPs could become even larger ${ }^{[20]}$, which indicates a better performance to some extent in their composites. Besides, Co NPs have better 
oxidation-resistance than Fe NPs although the later has a higher saturation magnetization ( 213 emu/g). Thus PPy/Co PNCs can be expected to have wide applications in the future.

There are at least four methods to prepare PPy NPCs, including simple blending, in situ polymerization, one-step chemical method, and electrochemical deposition ${ }^{[21]}$. The first three methods are reported frequently, yet they are technically possible to produce PPy NPCs powders only ${ }^{[9-19]}$. Considering that the PPy PNCs hardly dissolve in any inorganic or organic solvents, they would be used inconveniently in most situations where PPy PNCs are supposed to form films or coat upon machines' surface. By contrast, electrochemical processing could directly deposit a large area of PPy film on metal surface uniformly, which makes it one reliable method to use PPy NPCs industrially. Among those relatively fewer reports, Watanabe et al. have successfully prepared Co nano-clusters embedded in the PPy matrix by two-step electrodeposition, which firstly polymerized PPy films at a positive potential and then reduced Co nano-clusters at a negative potential ${ }^{[22]}$. This kind of PPy/Co PNCs indeed combined the characteristics of parent constituents, nonetheless the Co nano-clusters could only stay on the surface of the PPy and disperse unevenly. Therefore, other synthesis process needs to be developed to prepare PPy/Co NPCs with improved properties. Here, we present a novel electrochemical process to prepare PPy films embedded with Co NPs and the mechanism of electropolymerization process is explained. The composite exhibits multifunctional properties.

\section{Experiment}

All reagents are of analytical grade. Pyrrole is purified by vacuum distillation method before using. Other chemicals are not further purified. The synthesis process includes two steps as below.

( i) Preparation of water dispersible Co NPs: Firstly, the monodispersed Co NPs were 
synthesized by the chemical method of decomposing $\mathrm{Co}_{2}(\mathrm{CO})_{8}$ in phenyl ether ${ }^{[23]}$. The Co NPs were dispersed in $10 \mathrm{~mL}$ of hexane after being purified for two times. Mercaptoacetic acid $\left(\mathrm{C}_{2} \mathrm{H}_{4} \mathrm{O}_{2} \mathrm{~S}\right)$ was dropped into the dispersion of Co NPs until Co NPs were observed to agglomerate gradually and deposit from the solution. The dispersion was centrifuged and the precipitate was re-dispersed in $5 \mathrm{~mL}$ of deionized water.

(ii) Electropolymerization of PPy films embedded with Co NPs: All electropolymerizations were performed at room temperature in a conventional three-electrode cell with a Pt counter electrode, a saturated calomel electrode (SCE) and Pt-coated silicon substrates as the work electrode, which has been reported in our previous work ${ }^{[24]}$. This electropolymerization process was developed by modifying the method of preparing PPy ${ }^{[25]}$. The typical electrolyte was composed of $0.1 \mathrm{M}$ sodium sulfate, $0.001 \mathrm{M}$ sodium dodecylbenzenesulfonate, and $0.1 \mathrm{M}$ pyrrole while the $\mathrm{pH}$ value was adjusted to 3 by adding sulfuric acid. The electropolymerization of PPy/ Co PNCs achieves at a lower $\mathrm{pH}$ value than that of pure PPy. In order to reduce the time of Co NPs staying in acidic solution, several drops of well-dispersed Co NPs suspension, such as 6, 26, 50 and 100 drops, should be added into the electrolyte after all other experimental procedures were ready. After stirring the mixture for about $1 \mathrm{~min}$, electropolymerization started at once. One drop of Co NPs suspension contained about $0.6 \mu \mathrm{g}$ of Co NPs. The electropolymerization was carried out by cyclic voltammetry for 15 successive cycles. The potential was applied in the range of $+0.5 \mathrm{~V}$ to $+1.0 \mathrm{~V}$ and the scan rate was $25 \mathrm{mV} / \mathrm{s}$. The films were washed by deionized water several times and dried in the air.

The surface morphology of the films were examined by using a LEO-1450 scanning electron micro-scope (SEM).The chemical structures were analyzed by NICOLET iS10 Fourier transform 
infrared spectra (FTIR) and Renishaw inVia Raman spectra. The electrochemical tests of the films were carried out on an LK98BII microcomputer-based electro-chemical analyzer. Magnetic and electronic properties of the films were studied by a physical property measurement system (PPMS) at room temperature. The dielectric property was measured by a TH2828S impedance analyzer.

\section{Results and discussion}

Pure PPy can be prepared by anodic electropolymerization generally. Dodecylbenzenesulfonate anions can enter PPy as dopant during the electropolymerization and help PPy to obtain conductivity ${ }^{[26]}$. Different from pure PPy, PPy/Co PNCs were electropolymerized by cyclic voltammetry with potentials from $+0.5 \mathrm{~V}$ to $+1.0 \mathrm{~V}$. As shown in Fig. 1a, when Co NPs are added into the electrolyte solution, the hydrophilic group on the surface of Co NPs will have interaction with the amine group of pyrrole ring. It can be expected that more pyrrole molecules tend to absorb on Co NPs, which is similar with the absorption of 3-thiophene-acetic-acid on magnetite NPs ${ }^{[27]}$. Then when the electropolymerization of PPy takes place, Co NPs will exist spontaneously in the PPy films and form the PPy/Co PNCs. In the cyclic voltammetry method, those PPy films polymerized in later cycles would cover upon the former layers, gradually forming a layer-by-layer structure (Fig. 1b), and so that the Co NPs were completely encapsulated inside the PPy films. In fact, a DC potentiostatic method has ever been attempted to prepare PPy/Co PNCs. However, it is found that the PPy films would never polymerize upon the Pt substrate when more than six drops of the Co NPs dispersion were added in the electrolyte, no matter how positive the potential was. It may be attributed to that more pyrrole molecules absorbed on Co NPs electropolymerize directly upon Pt substrates at the initial stage because the rate of electropolymerization is fast at high potential. This kind of films 
probably has a poorer absorption capacity upon Pt substrates than pure PPy films, which results in the failure of further electropolymerization. Yet when cyclic voltammetry is used, the rate of polymerization at the initial stage will not be so fast, leaving enough time for the PPy films to adsorb on the Pt substrate. Besides, as shown in Fig. $2 \mathrm{a}-\mathrm{d}$, the surfaces of each PPy/Co films are all very compact, which is different from other layered films electropolymerized by potentiostatic method ${ }^{[27]}$, suggesting that these films have reliable high-quality. Compact microstructure of the PPy/ Co films is probably related to the uniform distribution of Co NPs with small sizes in the films. Uniform distribution of Co NPs in the PPy/Co films has been proved by XPS in our previous work $^{[24]}$.

The information of chemical bonds between the PPy films and the Co NPs was investigated by Fourier transform infrared (FT-IR) spectroscopy and Raman spectroscopy, as shown in Fig. 3 and Fig. 4. The inset in Fig. 3 is the FT-IR spectra for the pure Co NPs, on which the peak around the wave number of $300 \mathrm{~cm}^{-1}$ is due to the vibration of Co-O bonds. It means that a few Co atoms on the surface of Co NPs have been oxidized. Obviously, it is inevitable for the Co NPs to be partly oxidized when they are exposed to air, since $\mathrm{Co}^{2+} / \mathrm{Co}$ has a low standard electrode potential of $-0.282 \mathrm{~V}$ despite they are anchored by some organic surfactants. For the pure PPy films in Fig. 3, the peaks at $1611 \mathrm{~cm}^{-1}$ and $1400 \mathrm{~cm}^{-1}$ are due to the $\mathrm{C}=\mathrm{C}$ and $\mathrm{C}-\mathrm{N}$ stretching vibration of pyrrole rings, respectively ${ }^{[2,5]}$. The peak near $1128 \mathrm{~cm}^{-1}$ is due to the $\mathrm{C}-\mathrm{C}$ vibration ${ }^{[22]}$. The peaks at $1253 \mathrm{~cm}^{-1}$ and $1046 \mathrm{~cm}^{-1}$ are attributed to the $\mathrm{C}-\mathrm{H}$ in-plane and out-of-plane deformation vibration, respectively ${ }^{[28]}$. The small bond located at $927 \mathrm{~cm}^{-1}$ is due to the $\mathrm{C}$-C out-of-plane deformation vibration ${ }^{[24]}$. Most of these characteristic peaks are also observed on the FT-IR spectra of the PPy/Co PNCs although there are slight shifts compared to those of pure PPy, 
indicating the Co NPs are indeed embedded inside the PPy backbones and therefore influence the structure of PPy ${ }^{[29]}$. It is notable that the peak at $1400 \mathrm{~cm}^{-1}$ disappears in the PPy/Co PNCs, which means there is interaction between the Co NPs and the pyrrole ring's amine group, forming chemical bonds to some extent ${ }^{[22]}$. Fig. 4 shows the Raman spectra of the pure PPy film and the PPy/Co PNCs. The peak at $1570 \mathrm{~cm}^{-1}$ is due to the $\mathrm{C}=\mathrm{C}$ stretching while the peaks at $1322 \mathrm{~cm}^{-1}$ and $1412 \mathrm{~cm}^{-1}$ are due to $\mathrm{C}-\mathrm{C}$ in-ring and C-C inter-ring stretching, respectively. The peak around $1044 \mathrm{~cm}^{-1}$ is reflected to the symmetrical $\mathrm{CH}$ in-plane bending. The peaks at 935 and $974 \mathrm{~cm}^{-1}$ are due to the ring deformation. However, on the Raman spectroscopy of the PPy/Co PNCs, the peak at $935 \mathrm{~cm}^{-1}$ becomes pretty weak, which confirms the interaction between the Co NPs and the PPy amine group ${ }^{[30]}$.

The magnetic properties of the PPy films with different content of Co NPs were measured by physical property measurement system (PPMS) at room temperature. Our previous study shows that the pure PPy film presents an extremely weak intrinsic magnetism. When Co NPs are incorporated into PPy, the magnetic properties of the films will be improved greatly. In Fig. 5, it can be observed that with the increasing content of Co NPs, the saturation magnetization $\left(\mathrm{M}_{\mathrm{s}}\right)$ of the PPy/Co PNCs increases gradually. This directly proves that the more the Co NPs were added into the electrolyte, the larger Co content the PPy/Co PNCs have. Therefore one can control the magnetic properties of the PPy/Co PNCs by adjusting the content of Co NPs in the electrolyte, which is convenient in actual industry. Yet, the largest $M_{s}$ (about $0.4 \mathrm{emu} / \mathrm{g}$ ) is still lower than the pure Co NPs $(2.6 \mathrm{emu} / \mathrm{g})$. This might result from still relatively low content of Co NPs in the PNCs. Furthermore, the $M_{r} / M_{s}$ ratio $\left(M_{r}\right.$ is the remanence magnetization) is less than 0.1 for all the PNCs, which indicates a superparamagnetic property. The superparamagnetic critical size for 
Co NPs is below $6.7 \mathrm{~nm}{ }^{[31]}$. Since our Co NPs prepared chemically are observed to have a size of about $5.7 \mathrm{~nm}$ from the TEM image (Fig. 1c), it is clear that the Co NPs are dispersed very well inside the PPy without any aggregation.

The conducting property of PPy molecules originates from their delocalized $\pi$-bond structure and could be affected by Co NPs because the magnetism of Co comes from their d-electrons. The electrical conductivity of our samples was measured at room temperature. The voltage was applied between the Pt top and bottom electrodes. The Pt top electrode was deposited by DC magnetron sputtering at room temperature with a shadow mask and the Pt substrate serves as the bottom electrode. From Fig. 6a, the PPy/Co PNCs show semiconductor-like conductive behavior with a conductivity of $1.28 \times 10^{-5} \mathrm{~S} / \mathrm{m}$. The reason is that the Co NPs and dodecylbenzenesulfonate inside the films affect the charge transport based on delocalized $\pi$-bond structure. Further, because the PPy/Co PNCs are magnetic, their magnetoresistances (MRs) were also measured by four-point probe method at room temperature under 5000 Gauss external magnetic field. Since the magnetic field was not large enough to saturate MR, the PPy films with 26 drops of Co NPs show only a very low MR of $0.1 \%$, as shown in Fig. 6b. However it is clear that the PNCs exhibit negative MR under the magnetic field, which reflects the MR is induced by Co NPs because the pure PPy sample is supposed to show a positive MR value ${ }^{[17]}$.

The dielectric properties of the PPy/Co PNCs were measured by an AC impedance analyzer (TH2828S). As shown in Fig. 7, the real permittivity $\left(\varepsilon^{\prime}\right)$, imaginary permittivity $\left(\varepsilon^{\prime \prime}\right)$, and dielectric loss tangent $\left(\tan \mathrm{D}=\varepsilon^{\prime \prime} / \varepsilon^{\prime}\right)$ are dependent on frequency at room temperature. Both $\varepsilon^{\prime}$ and $\varepsilon^{\prime \prime}$ values of the PPy/Co PNCs switch from negative to positive values with the increasing frequency. This phenomenon is derived from the interfacial polarization ${ }^{[1]}$, where the charge 
carriers are accumulated at the internal interfaces arising from the Maxwell-Wagner-Sillars polarization effect ${ }^{[17]}$. The decrease of $\varepsilon^{\prime}$ at high frequency is induced by the dielectric relaxation ${ }^{[32]}$, which is usually caused by the delay of molecular polarization in an external alternative electric field ${ }^{[33]}$. The values of $\varepsilon^{\prime}$ and $\varepsilon^{\prime \prime}$ are both negative and high $\left(\left|\varepsilon^{\prime}\right|\right.$ or $\left|\varepsilon^{\prime \prime}\right|$ could be larger than $10^{11}$ ) at the low frequency, which can result in the reflection of the electromagnetic wave at the surface of the PPy/Co PNCs rather than normal refraction, suggesting a good electromagnetic shielding ability. On the other hand, the tan D has some peaks due to the resonance effect, in which the system tends to oscillate with greater amplitude than others at certain frequencies ${ }^{[34]}$. To be impressive, the PPy/Co PNCs present a remarkablely low dielectric loss ( $\tan \mathrm{D}<300$ ), especially at the high frequency, which might be used in the microelectronic devices with a very low energy loss.

\section{Conclusion}

We have prepared the PPy PNCs embedded with Co NPs by a novel electropolymerization method. The mechanism of the formation process and the microstructure of the PNCs have been carefully studied. The multifunctional PPy/Co PNCs show superparamagnetism, semiconductive transportation, special dielectrical property and MR at room temperature. The unique dielectric performance originates from the interaction between Co NPs and PPy backbones, which might be very useful, combined with their tunable magnetic properties, in the fields of electromagnetic shielding and data storage.

\section{Acknowledgement}

This work was partially supported by the National Basic Research Program of China (Grant No. 2015CB921502, 2012CB932702), the National Science Foundation of China (Grant Nos. 
61471036, 11174031, 51371024, 51325101, 51271020), NCET, PCSIRT, the Fundamental

Research Funds for the Central Universities and the State Key Lab of Advanced Metals and Materials (2014-Z01).

\section{Reference}

[1] J. Zhu, X. Zhang, N. Haldolaarachchige, Q. Wang, Z. Luo, J. Ryu, D. P. Young, S. Wei, Z. Guo, Polypyrrole metacomposites with different carbon nanostructures, J. Mater. Chem. 2012, 22, 4996.

[2] P. Mavinakuli, S. Wei, Q. Wang, A. B. Karki, S. Dhage, Z. Wang, D. P. Zhu, Z. J. Young, Z. Guo, Polypyrrole/silicon carbide nanocomposites with tunable electrical conductivity, J. Phys. Chem. C 2010, 114, 3874.

[3] J. Zhu, S. Wei, L. Zhang, Y. Mao, J. Ryu, N. Haldolaarachchige, D. P. Young, Z. Guo, Polyaniline-tungsten oxide metacomposites with tunable electronic properties, J. Mater. Chem. 2011, 21, 3952.

[4] H. Wei, X. Yan, Y. Li, S. Wu, A. Wang, S. Wei, Z. Guo, Electrochromic poly(DNTD)/WO 3 nanocomposite films via electorpolymerization, J. Phys. Chem. C 2012, 116, 4500.

[5] S. Wei, P. Mavinakuli, Q. Wang, D. Chen, R. Asapu, Y. Mao, N. Haldolaarachchige, D. P. Young, Z. Guo, Polypyrrole-titania nanocomposites derived from different oxidants, $J$. Electrochem. Soc. 2011, 158, 205.

[6] Z. B. Zha, X. L. Yue, Q. S. Ren, Z. F. Dai, Uniform polypyrrole nanoparticles with high photothermal conversion efficiency for photothermal ablation of cancer cells, Adv. Mater. 2013, 25,777 .

[7] L. F. Q. P. Marchesi, F. R. de Paula, A. J. A. de Oliveira, E. C. Pereira, Magnetic properties of 
polypyrrole doped with iron, Mol. Cryst. Liq. Cryst. 2010, 522, 301.

[8] H. Y. Yang, W. Jiang, Y. Lu, Fabrication and characteristic of conductive and ferromagnetic hollow composite microcapsules, Mater. Lett. 2007, 61, 1439.

[9] W. Z. Li, T. Qiu, L. L. Wang, S. S. Ren, J. R. Zhang, L. F. He, X. Y. Li, Preparation and electromagnetic properties of core/Shell polystyrene@polypyrrole@nickel composite microspheres, ACS Appl. Mater. Interfaces 2013, 5, 883.

[10] Y. Q. Wang, B. F. Zou, T. Gao, X. P. Wu, S. Y. Loua, S. M. Zhou, Synthesis of orange-like $\mathrm{Fe}_{3} \mathrm{O}_{4} / \mathrm{PPy}$ composite microspheres and their excellent $\mathrm{Cr}(\mathrm{VI})$ ion removal properties, J. Mater. Chem. 2012, 22, 9034.

[11] H. Zhang, X. Zhong, J. J. Xu, H. Y. Chen, $\mathrm{Fe}_{3} \mathrm{O}_{4} /$ Polypyrrole/Au nanocomposites with core/shell/shell structure: synthesis, characterization, and their electrochemical properties, Langmuir 2008, 24, 13748.

[12] L. Fang, T. Y. Dai, Y. Lu, Electromagnetic composite films based on polypyrrole hydro-sponge and $\mathrm{Fe}_{3} \mathrm{O}_{4}$ ferrofluid, Synth. Met. 2009, 159, 2101.

[13] L. G. Gai, X. Y. Han, Y. H. Hou, J. Chen, H. H. Jiang, X. C. Chen, Surfactant-free synthesis of $\mathrm{Fe}_{3} \mathrm{O}_{4} @ \mathrm{PANI}$ and $\mathrm{Fe}_{3} \mathrm{O}_{4} @ \mathrm{PPy}$ microspheres as adsorbents for isolation of PCR-ready DNA, Dalton Trans. 2013, 42, 1820.

[14] Q. Gao, D. Luo, M. Bai, Z. W. Chen, Y. Q. Feng, Rapid determination of estrogens in milk samples based on magnetite nanoparticles/polypyrrole magnetic solid-phase extraction coupled with liquid chromatography-tandem mass spectrometry, J. Agric. Food Chem. 2011, 59, 8543.

[15] H. Ozkazanc, S. Zor, Electrochemical synthesis of polypyrrole (PPy) and PPy vertical bar metal composites on copper electrode and investigation of their anticorrosive properties, Prog. 
Org. Coat. 2013, 76, 720.

[16] M. Chipara, R. Skomski, D. J. Sellmyer, Electrodeposition and magnetic properties of polypyrrole-Fe nanocomposites, Mater. Lett. 2007, 61, 2412.

[17] J. Guo, H. B. Gu, H. G. Wei, Q. Y. Zhang, N. Haldolaarachchige, Y. Li, D. P. Young, S. Y. Wei, Z. H. Guo, Silica doped nanopolyaniline with endured electrochemical energy storage and the magnetic field Effects, J. Phys. Chem. C 2013, 117, 10191.

[18] J. Azadmanjiri, K. Suzuki, C. Selomulya, A. Amiet, J. D. Cashion, G. P. Simon, The use of plasma treatment for simultaneous carbonization and reduction of iron oxide/polypyrrole core/shell nanoparticles, J. Nanopart. Res. 2012, 14, 1078.

[19] Y. Wang, Y. Huang, Q. F. Wang, Q. He, L. Chen, Preparation and electromagnetic properties of Polyaniline(polypyrrole)- $\mathrm{BaFe}_{12} \mathrm{O}_{19} / \mathrm{Ni}_{0.8} \mathrm{Zn}_{0.2} \mathrm{Fe}_{2} \mathrm{O}_{4}$ ferrite nanocomposites, Appl. Surf. Sci. 2012, 259, 486.

[20] J. P. Chen, C. M. Sorensen, K. J. Klabunde, Enhanced magnetization of nanoscale colloidal cobalt particles, Phys. Rev. B 1995, 51, 11527.

[21] H. M. Xiao, S. Y. Fu, Synthesis and physical properties of electromagnetic polypyrrole composites via addition of magnetic crystals, Cryst. Eng. Comm. 2014, 16, 2097.

[22] N. Watanabe, J. Morais, S. B. B. Accione, A. Morrone, J. E. Schmidt, M. C. M. Alves, Electronic, structural, and magnetic properties of cobalt aggregates embedded in polypyrrole, $J$. Phys. Chem. B 2004, 108, 4013.

[23] H. T. Yang, C. M. Shen, Y. K. Su, T. Z. Yang, and H. J. Gao, Self-assembly and magnetic property of colbalt nanoparticles, Appl. Phys. Lett. 2003, 82 (26), 4729.

[24] Z. D. Xu, M. Gao, L. N. Yu, L. Y. Lu, X. G. Xu, Y. Jiang, Co nanoparticles induced resistive 
switching and magnetism for the electrochemically deposited polypyrrole composite films, ACS Appl. Mater. Interfaces 2014, 6, 17823.

[25] N. Alizadeh, M. Mahmodian, a new dodecylsulfate ion-selective sensor based on electrochemically prepare polypyrrole and PVC, Electroanalysis, 2000, 12, 509.

[26] Mária Omastová, Miroslava Trchová, Jana Kovářová, Jaroslav Stejskal, Synthesis and structural study of polypyrroles prepared in the presence of surfactants, Synth. Met. 2003, 138, $447-455$

[27] Csaba Janáky, Csaba Visy, Ottó Berkesi, and Etelka Tombácz, Conducting polymer-based electrode with magnetic behavior: electrochemical synthesis of poly (3-thiophene-acetic-acid) / magnetite nanocomposite thin layers, J. Phys. Chem. C 2009, 113(4), 1352-1358.

[28] J. Zhu, S. Wei, L. Zhang, Y. Mao, J. Ryu, P. Mavinakuli, A. B. Karki, D. P. Young, Z. Guo, Conductive polypyrrole/tungsten oxide metacomposites with negative permittivity, J. Phys. Chem. C 2010, 114, 16335.

[29] H. Gu, Y. Huang, X. Zhang, Q. Wang, J. Zhu, L. Shao, N. Haldolaarachchige, D. P. Young, S. Wei, Z. Guo, Magnetoresistive polyaniline-magnetite nanocomposites with negative dielectrical properties, Polymer 2012, 53, 801.

[30] X. X. Yuan, X. Zeng, H. J. Zhang, Z. F. Ma, C. Y. Wang, Improved performance of proton exchange membrane fuel cells with p-toluenesulfonic acid-doped Co-PPy/C as cathode Electrocatalyst, J. Am. Chem. Soc. 2010, 132, 1754.

[31] B. D. Cullity, Introduction to Magnetic Materials. Addison-Wesley, Menlo Park, CA, 1972.

[32] J. Zhu, H. Gu, Z. Luo, N. Haldolaarachige, D. P. Young, S. Wei, Z. Guo, Carbon nanostructure-derived polyaniline metacomposites: electrical, dielectric, and giant 
magnetoresistive properties, Langmuir 2012, 28, 10246.

[33] R. Rosenbaum, A. Milner, R. Haberkern, P. Haussler, E. Palm, T. Murphy, S. Hannahs, B.

Brandt, Magnetoresistance of an insulating quasicrystalline AlPdRe film in large magnetic fields, J. Phys. Condens. Matter 2001, 13, 3169.

[34] J. Yao, Z. Liu, Y. Liu, Y. Wang, C. Sun, G. Bartal, A. M. Stacy, X. Zhang, Optical negative refraction in bulk metamaterials of nanowires, Science 2008, 321, 930. 


\section{Figure Captions:}

Fig. 1 (a) Schematic diagram of the electropolymerization of PPy/ Co PNCs. (b) Sectional view of the PPy/Co films. (c) TEM image of the Co NPs. The inset in (b) is the cyclic voltammetry curve of the electropolymerization.

Fig. 2 SEM images of (a) the pure PPy film, (b) PPy + 6 Co, (c) PPy +50 Co, (d) PPy + 100 Co film. $(\mathrm{PPy}+\mathrm{X}$ Co represents the film electropolymerized from the electrolyte solution which was added with X drops of Co NPs suspension).

Fig. 3 FT-IR spectra of the pure PPy and PPy/Co PNCs. The inset is the FT-IR spectrum of Co NPs.

Fig. 4 Raman spectra of the pure PPy and PPy/Co PNCs.

Fig. 5 Hysteresis loops of the Co NPs, PPy + 6 Co film, PPy + 26 Co film and PPy +50 Co film.

Fig. 6 (a) Current-voltage curve of PPy/Co PNCs. (b) Magnetoresistance of the PPy +26 Co film.

Fig. 7 Dependence of the real permittivity $\left(\varepsilon^{\prime}\right)$, imaginary permittivity $\left(\varepsilon^{\prime \prime}\right)$, and dielectric loss tangent (the inset) of the PPy/Co PNCs on frequency. 
Fig. 1 (M. Gao et al.)

a

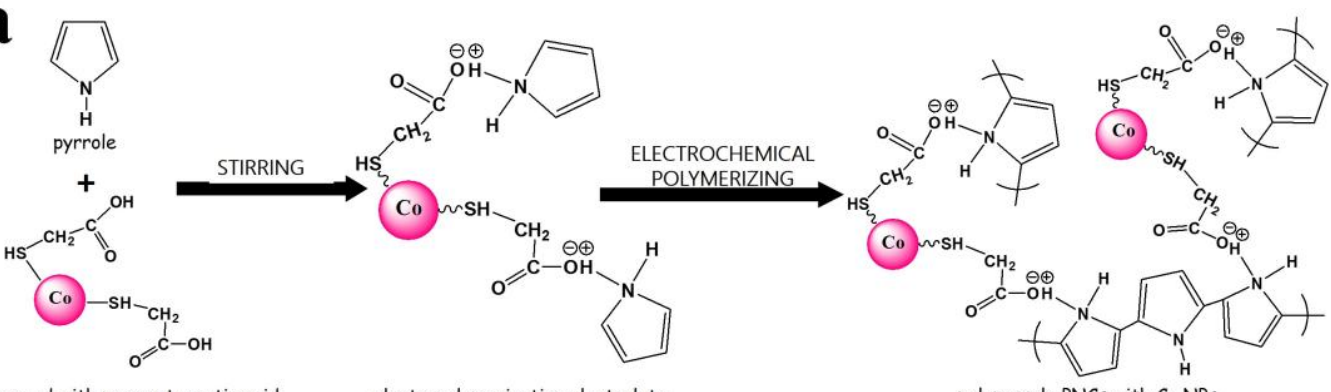

Co NPs capped with mercaptoacetic acid electropolymerization electrolyte
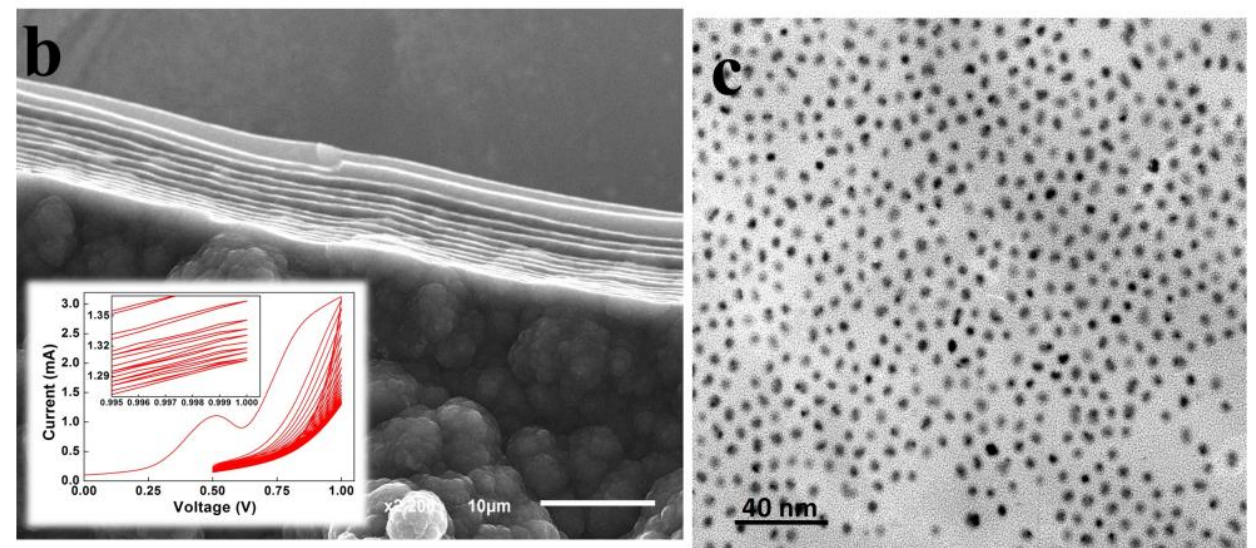

Fig. 2 (M. Gao et al.)

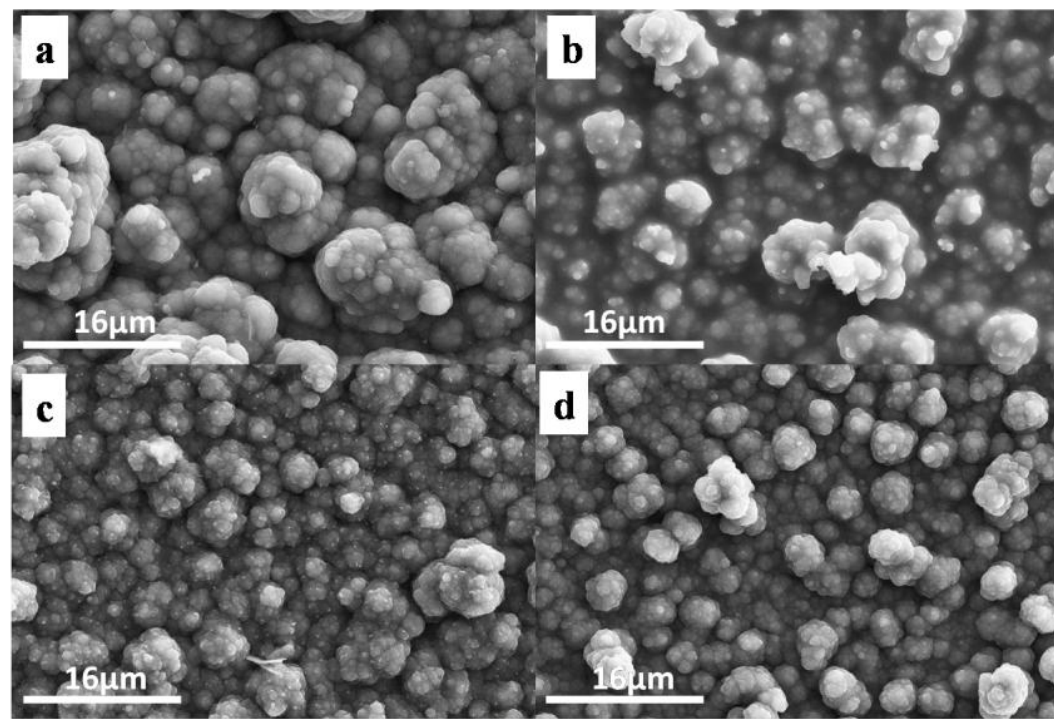


Fig. 3 (M. Gao et al.)

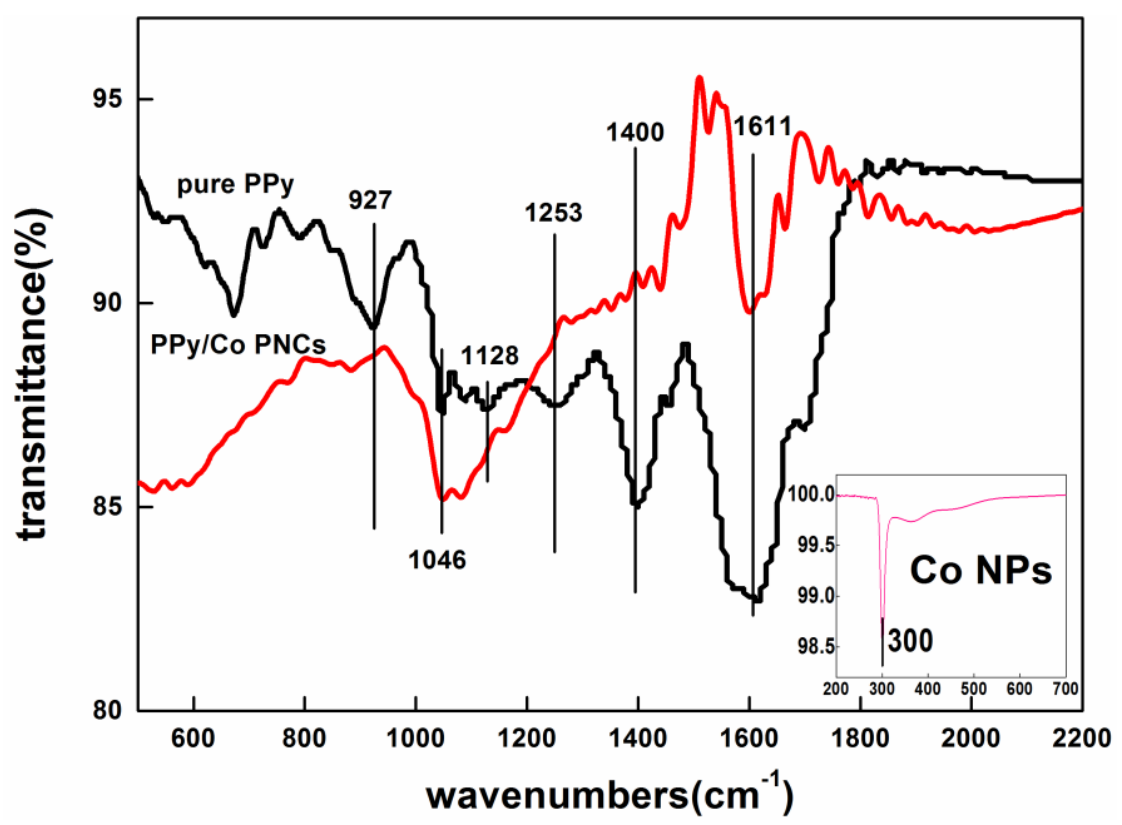

Fig. 4 (M. Gao et al.)

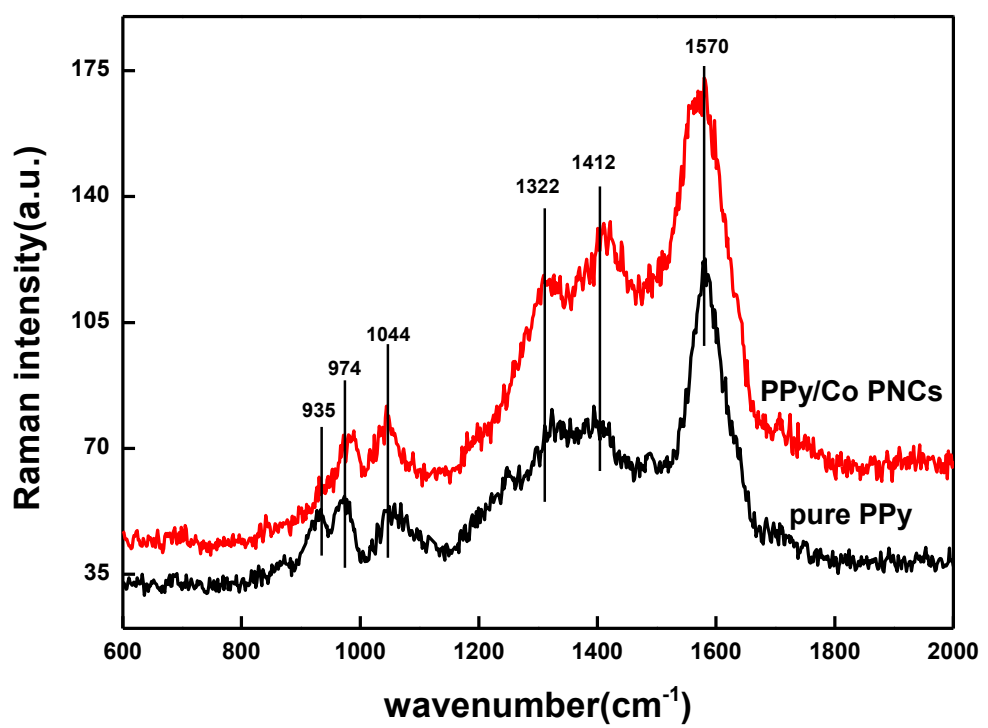


Fig. 5 (M. Gao et al.)
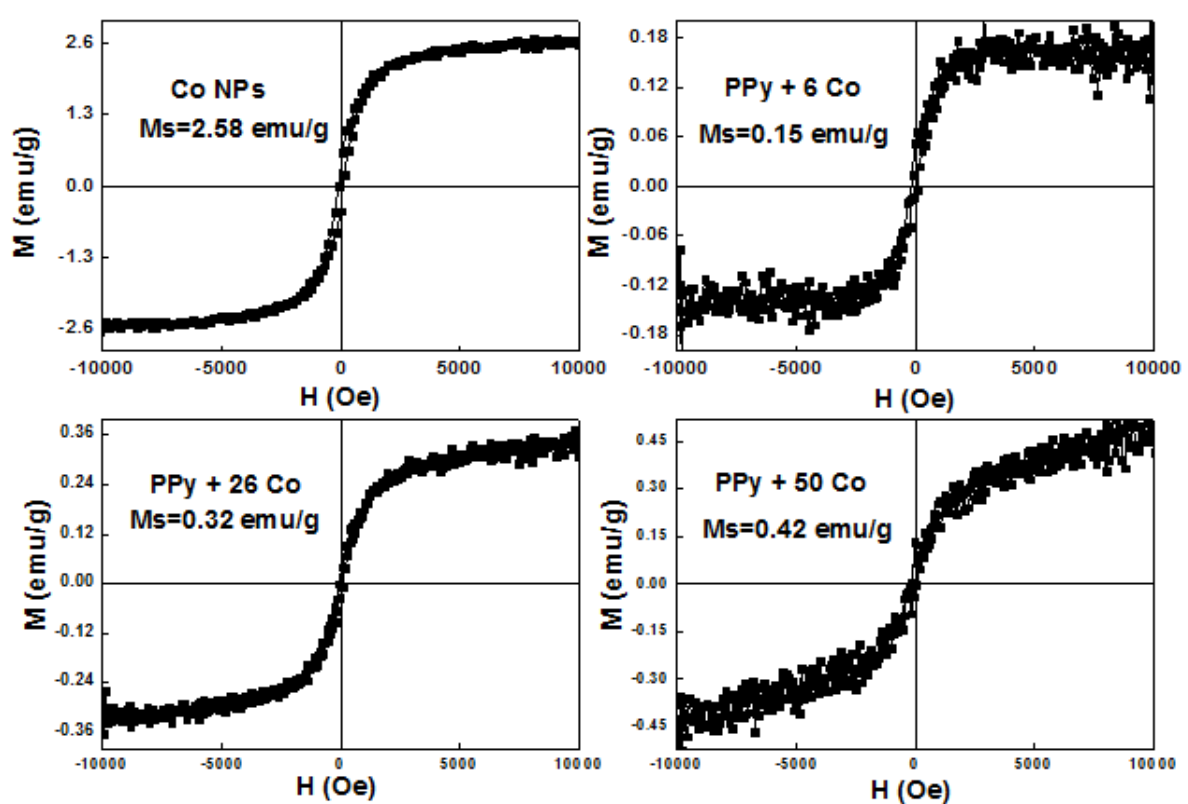

Fig. 6 (M. Gao et al.)
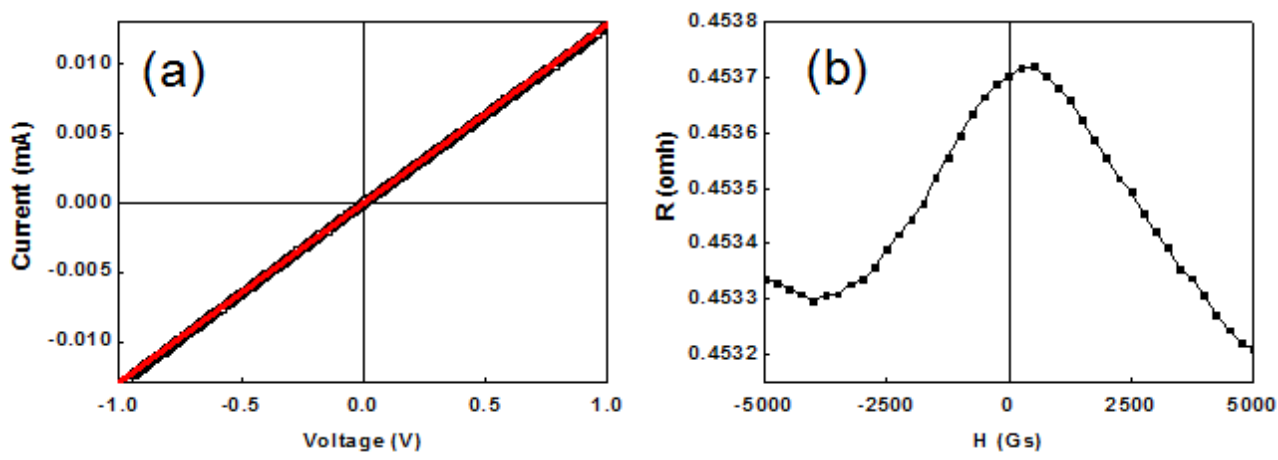
Fig. 7 (M. Gao et al.)

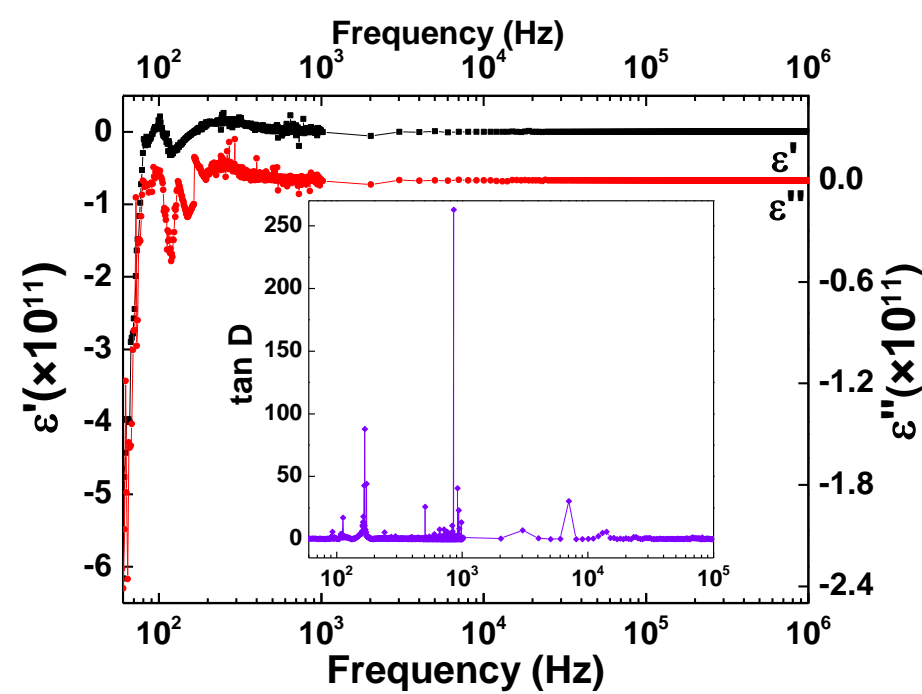

